\title{
Isolation and Functional Characterization of $M s F T a$, a FLOWERING LOCUS T Homolog from Alfalfa (Medicago sativa)
}

\author{
Junmei Kang ${ }^{\dagger}$, Tiejun Zhang ${ }^{\dagger}$, Tao Guo, Wang Ding, Ruicai Long, Qingchuan Yang \\ and Zhen Wang *(D) \\ Institute of Animal Science, Chinese Academy of Agricultural Sciences, Beijing 100193, China; \\ kangjunmei@caas.cn (J.K.); tiejunzhang@126.com (T.Z.); Yushen0002008@126.com (T.G.); \\ dingwang@126.com (W.D.); dragongodsgod@163.com (R.L.); qchyang66@163.com (Q.Y.) \\ * Correspondence: wangzhen@caas.cn; Tel.: +86-10-6281-6357 \\ + These authors contributed equally to this work.
}

Received: 24 February 2019; Accepted: 15 April 2019; Published: 22 April 2019 updates

\begin{abstract}
The production of hay and seeds of alfalfa, an important legume forage for the diary industry worldwide, is highly related to flowering time, which has been widely reported to be integrated by FLOWERING LOCUS T (FT). However, the function of $F T(\mathrm{~s})$ in alfalfa is largely unknown. Here, we identified $M s F T a$, an $F T$ ortholog in alfalfa, and characterized its role in flowering regulation. $M s F T a$ shares the conserved exon/intron structure of FTs, and the deduced MsFTa is $98 \%$ identical to MtFTa1 in Medicago trucatula. MsFTa was diurnally regulated with a peak before the dark period, and was preferentially expressed in leaves and floral buds. Transient expression of MsFTa-GFP fusion protein demonstrated its localization in the nucleus and cytoplasm. When ectopically expressed, MsFTa rescued the late-flowering phenotype of $f t$ mutants from Arabidopsis and M. trucatula. MsFTa over-expression plants of both Arabidopsis and M. truncatula flowered significantly earlier than the non-transgenic controls under long day conditions, indicating that exogenous MsFTa strongly accelerated flowering. Hence, $\mathrm{MsFTa}$ functions positively in flowering promotion, suggesting that MsFTa may encode a florigen that acts as a key regulator in the flowering pathway. This study provides an effective candidate gene for optimizing alfalfa flowering time by genetically manipulating the expression of MsFTa.
\end{abstract}

Keywords: alfalfa; MsFTa; flowering time; transgenic plants

\section{Introduction}

Flowering time, the switch from vegetative to reproductive growth, is a key trait for the seasonal and geographical adaptation of flowering plants. The mechanism of flowering is best understood in the annual plant Arabidopsis, in which the timely onset of flowering is regulated by an intricate network with various genetic regulators responding to endogenous (gibberellin, autonomous and aging) or environmental (vernalization and photoperiod) triggers, as reviewed in recent years [1-4]. The balance of signals from these pathways is integrated by a common set of genes, including FLOWERING LOCUS C (FLC)/MADS AFFECTING FLOWEIRNG (MAF), FLOWERING LOUCS T (FT), CONSTANS (CO) and SUPPRESSOR OF OVEREXPRESSION OF CONSTANS 1 (SOC1), to determine the flowering time [5-7]. In Medicago, however, FLC/MAF clade flowering repressors are missing and $C O$-like genes do not seem to regulate flowering [8-10]. Hence, florigen encoding gene $F T$, acting as a long-distance hormone signal between leaves and shoot apical meristems, is the most important flowering integrator of the legume species. 
Since the search for the flowering hormone florigen in the 1930s, an increasing number of FT-like genes have been identified in a wide range of annual plants, such as rice, soybean and barrel clover [11-13]. For example, in M. truncatula five FT-like genes, namely MtFTa1, MtFTa2, MtFTb1, $M t F T b 2$ and $M t F T c$, have been characterized. Among them, MtFTa1, MtFTb1 and MtFTc rescued the late flowering phenotype of Arabidopsis $f t-1$ mutant, while the other two genes failed to act as a florigen [11]. Functional differentiation among FT paralogs derived from lineage-specific duplication has been observed in several species, such as soybean, potato and rice [13-15]. The diversity of the functions of FT paralogs is attributed to the expression pattern shift and the divergence in gene regulatory networks.

In recent years, studies on $F T$-integrated flowering regulation have focused on economically important perennial plants including grasses (e.g., switchgrass and ryegrass) $[16,17]$ and trees (e.g., poplar, apple and cherry) [18-20]. In contrast to annual plants, FT is expressed in both leaves and flowers of several perennial species such as sweet cherry, kiwifruit and leafy spurge [21-23]. Functional coordination of perennial FT paralogs revealed the evolution of a complex perennial adaptive trait after genome duplication. For example, poplar FT1 determined the onset of reproductive stage in winter, whereas FT2 promoted vegetative growth and inhibited bud set in the growing season [18]. Ectopic over-expression of $F T$ perennial orthologs in Arabidopsis caused early flowering in most cases, suggesting a conserved function in perennial species [16-20,22].

Alfalfa ( $M$. sativa), one of the most important forages globally, exhibits seasonal flowering. Different from perennial woody plants, which are not able to form flower buds during the first several years of their life cycle, herbaceous alfalfa grows vegetatively at early developmental stages and then switches to the reproductive stage in the same year. In Northern China, a main alfalfa planting region in the country, the first cutting coincides with the rain season, which severely limits hay production. Breeding alfalfa cultivars with optimal flowering time would facilitate the improvement of the quantity and quality of alfalfa. Our study here demonstrated that $M s F T a$, the first isolated $F T$ from alfalfa, not only shared the conserved functional domains of its orthologs, but also acted ectopically in accelerating flowering. These findings would supply a potential candidate for the generation of alfalfa cultivars with an optimal flowering time for the main planting regions in Northern China via genetic modulation of MsFTa expression.

\section{Results}

\subsection{MsFTa Sequence Shared the Common Features of FT-like Genes}

FLOWERING LOCUS T (FT) genes have been intensively documented in many plants including $M$. truncatula, a model annual legume species [8-11]. Up till now, five MtFTs, namely MtFTa1, $M t F T a 2, M t F T b 1, M t F T b 2 \& M t F T c$, have been identified from $M$. truncatula. Using MtFTa1 as a query sequence, we did a BLAST search against the most updated $M$. truncatula genome database (http://plants.ensembl.org/Medicago_truncatula) and found that besides the four MtFT paralogs, one more gene (Mt6g033040) annotated as Flowering Locus Protein T (MtFT) was hit with a homology of $64-73 \%$ to its paralogous proteins (Table S1). Thus, the M. truncatula genome encodes at least six FT-like genes with sequence similarity higher than $60 \%$ (Figure 1). 


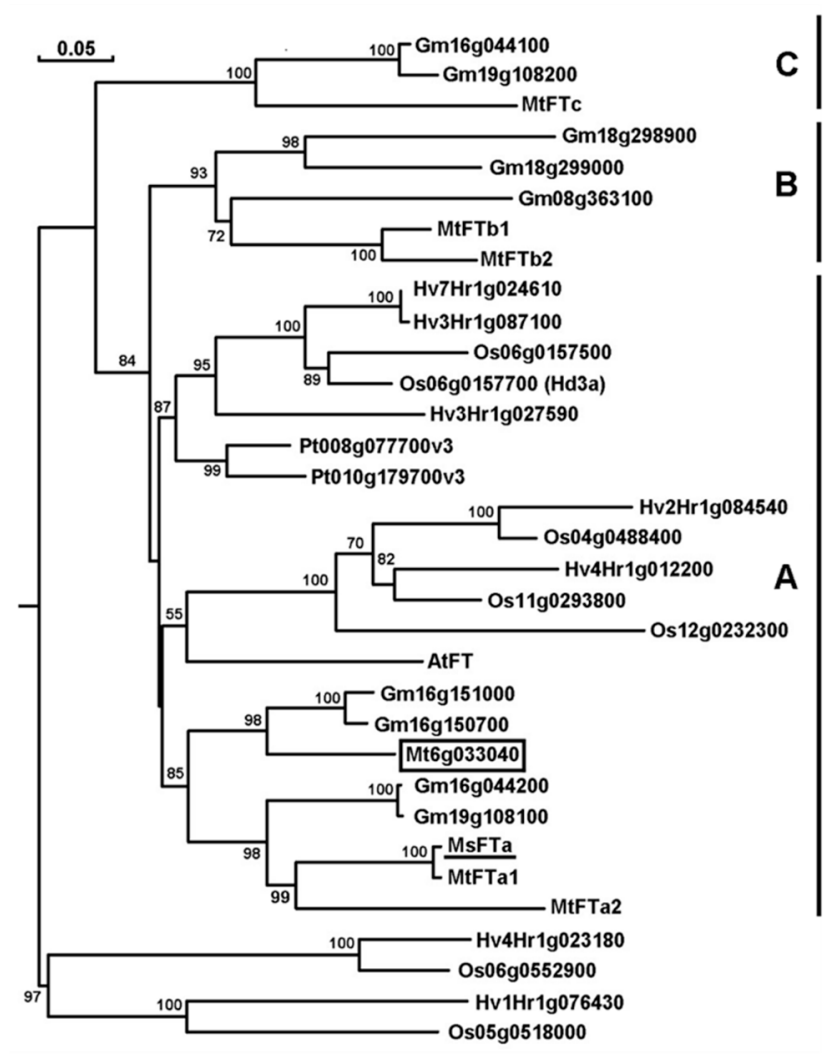

Figure 1. Phylogenetic analysis of MsFTa and its orthologs in the indicated plant species. The full-length sequence of $33 \mathrm{FT}$ and FT-like proteins from six model species was analyzed using DNAMAN (version 7.0). The numbers at the nodes of the neighbor-joining tree indicate the bootstrapping values based on 1000 interactions. MsFTa was underlined and Mt6g033440, a novel FT in M. truncatula was boxed. Gene accession numbers: MsFTa (GenBank: JF681135); Arabidopsis thaliana (At): AtFT (At1g65480); Medicago truncatula (Mt): MtFTa1 (Mt7g084970), MtFTa2 (Mt7g085020), MtFTb1 (Mt7g006630), MtFTb2 (Mt7g006690), MtFTc (Mt7g085040), MtFT (Mt6g033040); Glycine $\max (\mathrm{Gm}):$ Gm19g108100, Gm18g298900, Gm18g299000, Gm16g151000, Gm16g044200, Gm16g150700, Gm16g044100, Gm19g108200, Gm08g363100; Populus trichocarpa (Pt): Pt8g077700, Pt10g179700; Hordeum vulgare (Hv): Hv1Hr1g076430, Hv2Hr1g023180, Hv2Hr1g084540, Hv3Hr1g027590, Hv3Hr1g087100, Hv4Hr1g012200, Hv7Hr1g024610 and Oryza sativa (Os): OsHd3a (Os06g0157700), Os04g0488400, Os05g051800, Os06g0157500, Os06g0552900, Os11g0293800, Os12g0232300.

To investigate flowering regulation in perennial legume alfalfa, we cloned an FT-like cDNA from the forage by combination of RT-PCR and RACE strategy based on the sequence information of MtFTs. The 1029 bp cDNA sequence contained an open reading frame (ORF) of 531 bp (GenBank: JF681135) (Figure S1). The putative protein consists of 176 amino acids with theoretical isoelectric point (pI) of 8.23 and molecular weight of $19.7 \mathrm{kDa}$, respectively. Phylogenetic analysis of 33 FT and FT-like proteins from rice, barley, Arabidopsis, soybean, barrel clover and poplar demonstrated that the six MtFTs were grouped into three subclasses, namely Subclass A (MtFTa1, MtFTa2 \& Mt6g033040), Subclass B (MtFTb1 \& MtFTb2) and Subclass C (MtFTc) (Figure 1). The putative alfalfa FT protein was clustered into Subclass A sharing the same branch with MtFTa1 and MtFTa2 (Figure 1). Hence, it was designated as MsFTa, the first FLOWERING LOCUS T gene identified in alfalfa. Compared with Subclass A members from Arabidopsis, rice and barley, MsFTa was more closely related to the FTs of legume plants such as barrel clover and soybean (Figure 1). Sequence homology analysis showed that MsFTa shared an identity of 59-99\% with MtFT members, the lowest identity with MtFTc and the highest with MtFTa1, respectively (Table S1). The sequence similarity of MsFTa with FTs from the other five species ranges from $54 \%$ to $84 \%$ (Table S1). Multiple alignment revealed that, like its orthologs, 
MsFTa has a ligand-binding motif with a conserved key residue Tyr (Y) at position 85, an external loop of a 14-amino acid segment (residues 128-141) and a triad (residues 150-152) at the C-terminal end (Figure 2) [24-26]. In addition, MsFTa features a large number of alpha helices, beta-folds and random coil structures (Figure S2a). Our prediction using ScanProsite showed that most regions of MsFTa are hydrophilic (Figure S2b), indicating it is a hydrophilic protein.

Hv1Hr1g076430
Hv2Hr1g023180
Hv2Hr1g084540
Hv3Hr1g027590
Hv3Hr1g087100
Hv4Hr1g012200
Hv7Hr1g024610
Pt010g179700
Pt008g077700
Gm18g298900
Gm18g299000
Gm16g151000
Gm16g044200
Gm19g108100
Gm16g150700
Gm16g044100
Gm19g108200
Gm08g363100
Os04g0488400
Os05g0518000
Os06g0157500
Os06g0552900
Os11g0293800
Os12g0232300
Os06g0157700
AtFT
Mt6G033040
MtFTC
MtFTb2
MtFTb1
MtFTa2
MtFTa1
MsFTa



Hv1Hr 19076430 Hv2Hr1g023180 $\mathrm{Hv} 2 \mathrm{Hr} 1 \mathrm{~g} 084540$ Hv3Hr 19027590 Hv3Hr 19087100 Hv4Hr1g012200 $\mathrm{Hv} 7 \mathrm{Hr} 1 \mathrm{~g} 024610$ Pt010g179700 Pt008g077700 Gm18g298900 Gm18g299000 Gm16g151000 Gm16g044200 Gm19g108100 Gm16g150700 Gm16g044100 Gm 08 g 363100 Os04g0488400 Os05g0518000 Os 0690157500 Os06g0552900 Os11g0293800 Os12g0232300 Os06g015770 Mt6G033040 MtFTC MtFTb2 MtFTb1 MtFTa2 MtFTa1 MsFTa



-binding motif

External loop

$\overline{\mathrm{LYN}}$

Figure 2. Sequence alignment of FTs in the indicated species. Sequence was aligned using DNAMAN Version 7 (Lynnon Corporation, Quebec, Canada). Homology level was highlighted by shading in color: black for $100 \%$, pink for $\geq 75 \%$, blue for $\geq 50 \%$ and yellow for $\geq 33 \%$ identity. The ligand-binding motif and the external loop of 14 -amino acid stretch were underlined. The amino acid residues encoded by exon 2 ( $62 \mathrm{bp}$ ) and exon 3 (41 bp) were underlined with a dashed line. Triangles indicated the conserved amino acids in the ligand-binding pocket. An asterisk $\left(^{*}\right)$ indicated the conserved key residue Tyrosine (Y) at position 85 of MsFTa.

Comparison of the exon-intron structure of FT-like genes demonstrated that like most FT-like genes from a variety of species $[11,13,27,28]$, MsFTa is composed of four exons separated by three introns 
(Figure 3). Interestingly, exon 2 is universally $62 \mathrm{bp}$ and exon 3 is $41 \mathrm{bp}$, except two barley FT-like genes (Hv3Hr1g0871002 and Hv7Hr1g0246102) (Figure 3). The 2nd and 3rd exons of MsFTa encode the N-terminal of the ligand-binding motif, including the amino acids forming the ligand-binding pocket and the key residue Tyrosine (Y) at position 85 (Figure 2). Therefore, MsFTa not only has the same exon composition with conserved open reading frame but also possesses the FT functional domains shared by a wide range of plant species.
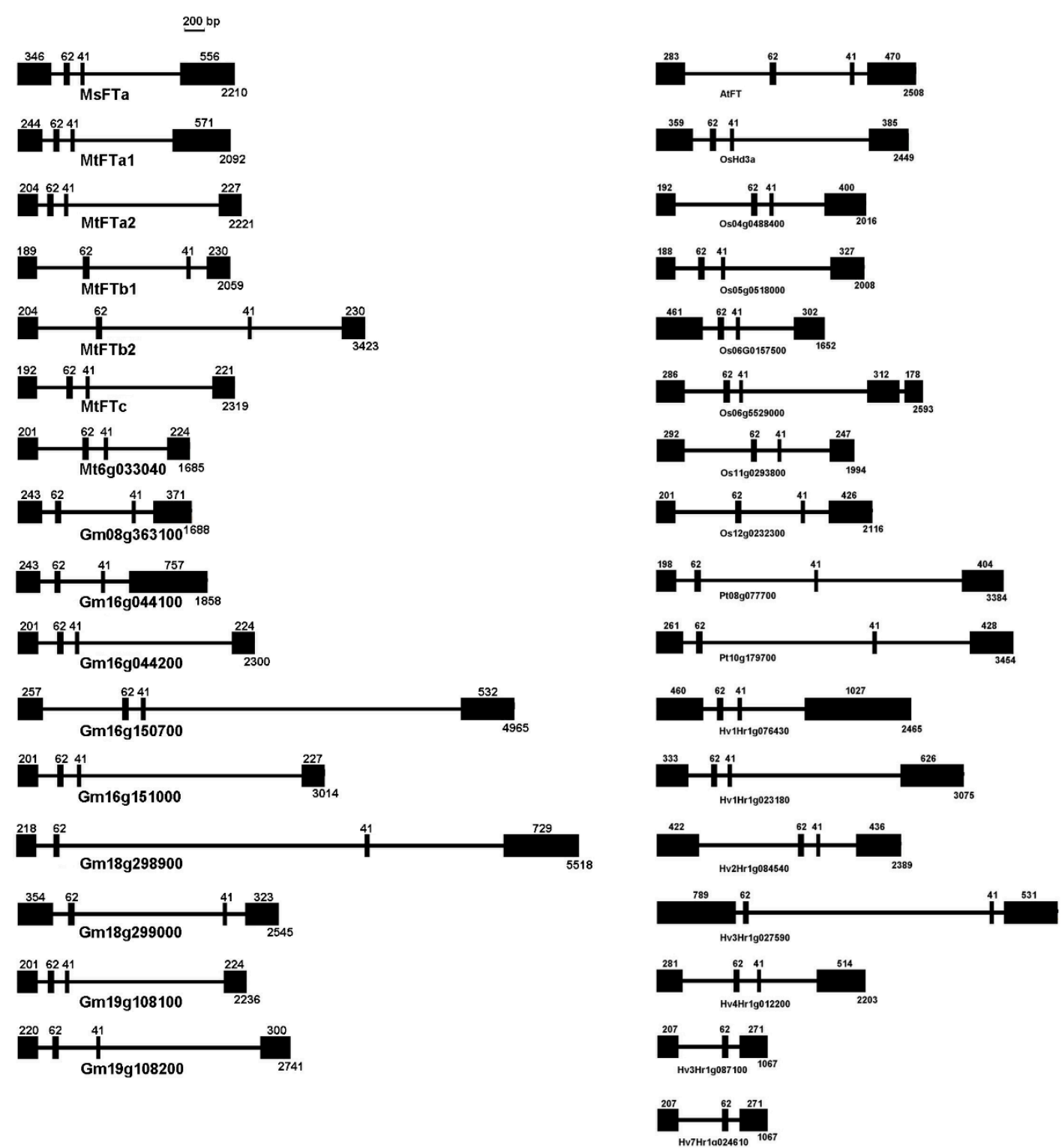

Figure 3. Schematic representation of the exon/intron structure of FTs in the indicated species. The information of FT gene composition is from EnsemblPlants (http://plants.ensembl.org). Gene structure was drawn using DNAMAN (Version 7). Solid lines represent introns and black boxes represent the exons with length indicated.

\subsection{The MsFTa-GFP Recombinant Protein Resided in Both the Cytoplasm and Nucleus}

To examine the subcellular localization of MsFTa, 35S::MsFTa-GFP and 35S::GFP were transformed into onion epidermal cells separately. The transient expression of the GFP control was uniformly distributed throughout the cell, whereas the MsFTa-GFP fusion protein was observed in the nucleus and cytoplasm, especially the membrane (Figure 4). This result is consistent with the recent observations of FT-like proteins in perennial species such as switchgrass and sweet cherry [16,22]. 


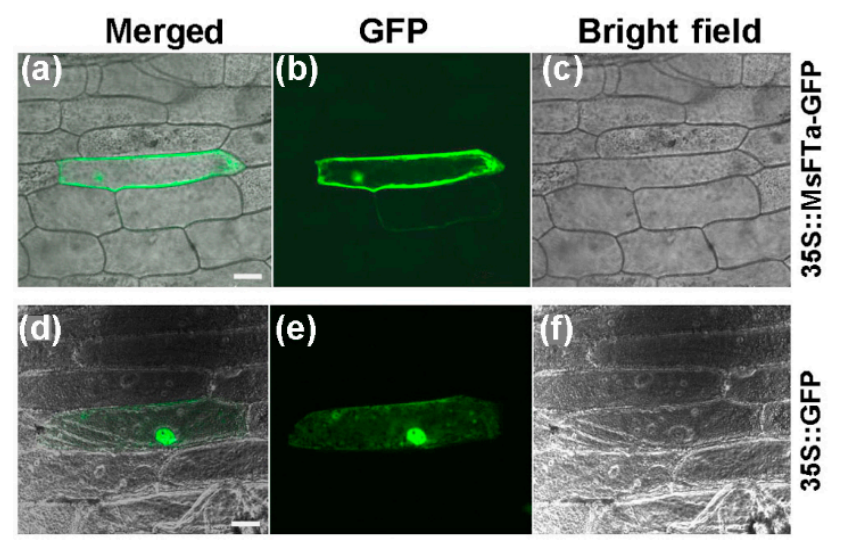

Figure 4. Subcellular localization of the MsFTa-GFP fusion protein in onion epidermal cells. The MsFTa-GFP fused construct and control vector pA7-GFP [29] were transiently transformed into onion epidermal cells by microprojectile bombardment. All images were captured using a confocal laser scanning microscope (Olympus FV500). (a-c): Image of onion epidermal cells expressing 35S::MsFTa-GFP taken under GFP fluorescence (b) or in bright field (c), the merged image was shown in (a); (d-f): Image of onion epidermal cells expressing 35S::GFP taken under GFP fluorescence (e) or in bright field (f), the merged one was shown in (d). Bar $=100 \mu \mathrm{m}$.

\subsection{Diurnal and Tissue-Specific Expression Pattern of MsFTa}

To monitor the temporal and spatial expression profiles of $M s F T a$, quantitative RT-PCR was performed using MsFTa-specific primers. Analysis of the MsFTa transcript demonstrated that the level of MsFTa progressively increased after $6 \mathrm{~h}$ light and reached a peak at $16 \mathrm{~h}$ light. The onset of darkness caused a sharp deduction of $\mathrm{MsFTa}$ and the descending trend continued during the night period (Figure 5a). Spatially, four tissues from both underground and aerial organs were tested. MsFTa was detected in all tested tissues including roots, stems, floral buds and leaves with the highest expression in leaves, which is approximately 3.4-fold of the amount in roots (Figure 5b). The expression pattern of $M s F T a$ is highly reminiscent of its legume homologs, especially MtFTa2 in M. truncatula [11]. Detailed analysis demonstrated that besides cauline leaves, a higher level of $F T$ transcript was detected in flowers, siliques, and seeds at early stages (http://bbc.botany.utoronto.ca/efp) (Figure S3).
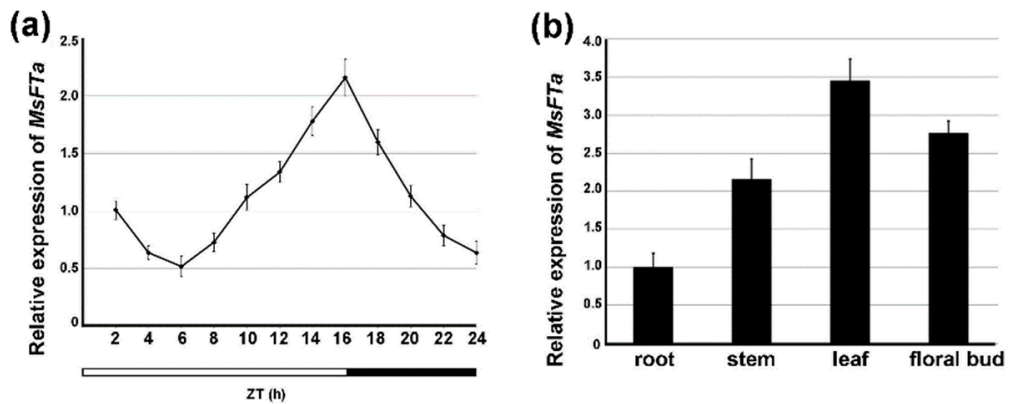

Figure 5. Diurnal and tissue-specific expression of MsFTa. (a) The expression level of MsFTa transcript during $24 \mathrm{~h}$ zeitgeber time (ZT) under LD. The white bar represents day time and the black bar represents night. (b) Relative expression of MsFTa in roots, stems, leaves and flowers of alfalfa at ZT 14. Bars represent the means \pm SD of three biological replicates.

\subsection{Ectopic Expression of MsFTa in Arabidopsis Strongly Accelerated Flowering}

To explore the biological function of MsFTa in modulating flowering, the construct of MsFTa driven by the cauliflower mosaic virus $35 \mathrm{~S}$ promoter was introduced separately into wild type Arabidopsis and $f t-10$, a T-DNA insertion mutant of $F T$, by agrobacterium infiltration. The existence of a 35S::MsFTa construct in two independent transgenic Arabidopsis T2 lines (T2-1 \& T2-4) with kanamycin 
resistance was confirmed by PCR (Figure 6a). Semi-quantitative RT-PCR of the over-expression lines demonstrated that MsFTa transcript was abundantly expressed in both transgenic lines while absent in wild type Arabidopsis (Figure 6b). Phenotypically, the over-expression plants exhibited early flowering (Figure 6c). The measurement of leaf number showed that wild type plants flowered with 15.4 leaves, whereas T2-1 and T2-4 over-expression lines flowered with 12.1 and 11.4 leaves, respectively $(p<0.05)$ (Figure 6d). Consistently, in terms of days after germination (DAG), the first flower of line T2-1 and T2-4 appeared at 16.1 and 17.8 DAG, respectively, while the first flower of the wild type was observed at 24.3 DAG $(p<0.05)$ (Figure 6e). These results suggested that flowering time of the transgenic Arabidopsis was associated with MsFTa transcript level.

(a)

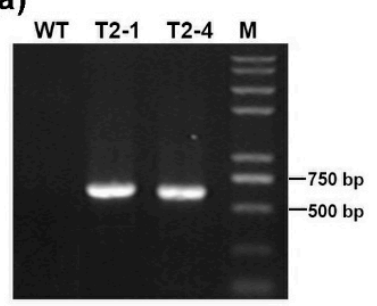

(b)

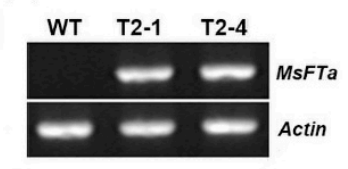

(c)

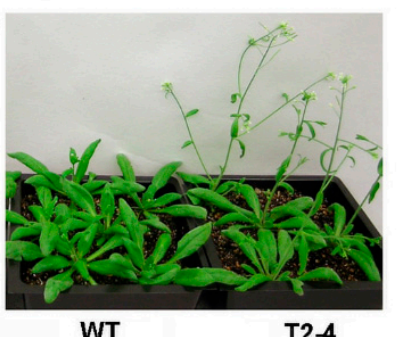

(d)

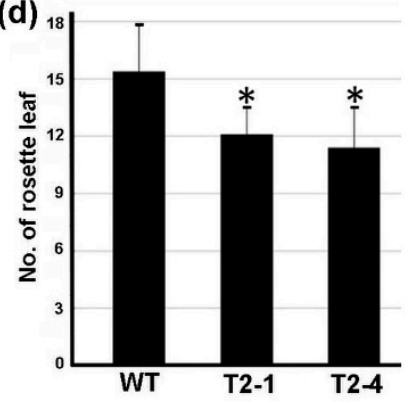

(e)

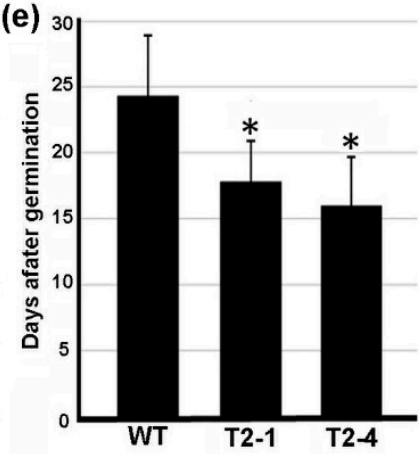

(f)

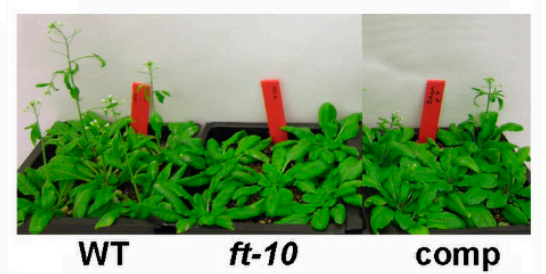

(g)

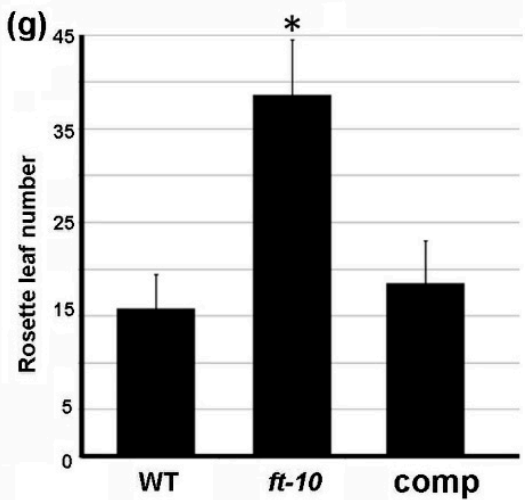

Figure 6. Ectopic expression of MsFTa in Arabidopsis caused early flowering. (a) Verification of two independent transgenic Arabidopsis lines (T2-1 and T2-4 of the T2 generation) harboring 35S::MsFTa construct by PCR. (b) Transcriptional analysis of MsFTa in the two transgenic lines verified in (a). Arabidopsis Actin 2 (AT3g18780) was used as internal control. Leaves from two-week old plants were harvested for total RNA extraction. (c) Three-week-old plants of Col-0 and MsFTa over-expressing line (T2-4). (d,e) Flowering time analysis of the over-expression T2 plants grown under LD conditions in terms of days after germination (d) and the No. of rosette leaves (e). At least 15 plants from three batches were scored for each line. The significant difference between the two genotypes was indicated as * (Student's t-test, $p<0.05$ ). (f) Four-week-old plants of Col-0, ft-10 and the transgenic $f t-10$ expressing 35S::MsFTa (comp), showing rescued flowering time. (g) Statistical analysis of the No. of rosette leaves upon the emergence of the first flower. * indicated the significant difference from wild type (Student's $t$-test, $p<0.05)$.

Arabidopsis mutant $f t-10$ is a strong allele showing late-flowering phenotype. Under LD, $f t-10$ flowered when the mutant plant had 36.8 leaves, while wild type flowered with 15.4 leaves $(p<0.05)$ (Figure 6f,g). For the transgenic plants in $f t-10$ background, eight out of 10 independent $\mathrm{T} 1$ lines flowered at a time similar to that of wild type. Flowering time analysis of a representative T2 line showed that the $M s F T a$ transgenic $f t-10$ flowered with 16.5 leaves (Figure $6 \mathrm{f}, \mathrm{g}$ ), indicating that introduction of $35 \mathrm{~S}:: \mathrm{MsFTa}$ complemented the late-flowering phenotype of $f t-10$ mutant. Except flowering time, the transgenic plants did not display obvious morphological difference. Taken together, our findings 
demonstrated that constitutive expression of alfalfa MsFTa in Arabidopsis resulted in early flowering, suggesting a positive role of $M s F T a$ in flowering regulation.

\subsection{Ectopic Expression of MsFTa Promoted Flowering in Transgenic M. truncatula Plants}

Given the close phylogenetic relationship and high sequence identity of MsFTa to MtFTa1, both barrel clover wild type R108 and mtfta1-1, a late-flowering mutant (NF3307) caused by the insertion of a Tnt1 retrotransposon in the first exon of MtFTa1 [11], were transformed with the construct of $35 \mathrm{~S}:: \mathrm{MsFTa}$ mentioned above. Flowering time of the transgenic plants was measured as days to produce the first flower under LD conditions. Compared with R108 which flowered at D56, the two independent T1 lines (T1-1 \& T1-2) overexpressing 35S:: MsFTa produced the first flower at D35 and D32, respectively. Over-expression of MsFTa in $M$. truncatula resulted in statistically significant early flowering $(p<0.05)$ (Figure 7a). For $m t f t 1-1$ plants expressing 35S::MsFTa, the flowering time of two independent transgenic lines was analyzed. As shown in Figure 7b, the first flower of the two lines emerged at D58 and D61, while fta1-1 flowered at D83, which is significant later than R108 at D56 $(p<0.05)$, indicating that $M s F T a$ successfully rescued the late flowering phenotype of $f t a 1-1$. These results suggested that similar to ectopic expression of MsFTa in Arabidopsis, introduction of MsFTa into M. truncatula caused strong flowering promotion.

(a)

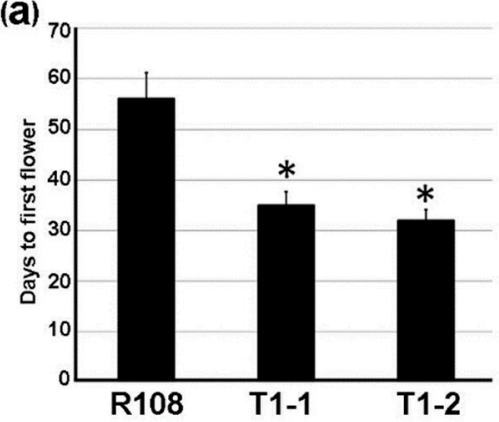

(b)

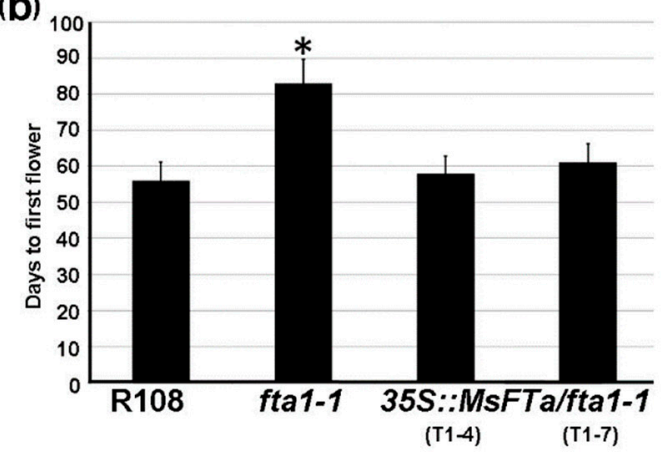

Figure 7. Ectopic expression of MsFTa in M. truncatula caused early flowering. (a) Comparison of flowering time between Medicago cv R108 overexpressing 35S::MsFTa (T1) and control (R108) plants under LD conditions. (b) Flowering time analysis of M. truncatula fta1-1 mutant (NF3307) plants ectopically expressing 35S::MsFTa (T1) under LD conditions. Flowering time was measured as days to the emergence of the first flower under LD conditions. Two independent lines for each transformation were analyzed. Data represent mean \pm SD of three biological replicates. * indicates a significant difference from wild type (Student's $t$-test, $p<0.05$ ).

\section{Discussion}

Precise flowering time ensures that the onset of flowering occurs at an ideal time to optimize plant biomass and fitness in specific environmental conditions. Although diverse upstream components of flowering pathways have been identified from plants, FLOWERING LOCUS T appears to be a universal regulator ultimately integrating flowering signaling in crops, vegetables, grass and trees $[11,18,26,30]$. In this study, we provided molecular and genetic evidence that $M s F T a$, an $F T$ homolog from alfalfa, functioned to accelerate flowering.

As a member of an ancient phosphatidylethanolamine-binding protein (PEBP) gene family, FT has a conserved exon/intron structure and highly identical protein sequence across species [13,31]. Indeed, our results demonstrated that MsFTa possessed the characteristic features of a classic FT gene. First, MsFTa, similar to $F T$-like genes, has four exons with conserved exon/intron boundaries $[13,27,28]$. Over-expression of the chimeric genes constructed by replacing individual exons of $F T$ revealed that substitution of the fourth exon, especially the external loop caused late flowering, presumably the crystal structure was affected due to the sequence variation of the exon [24]. It has been reported recently that 
exons two and three were also crucial for the functional conversion of FT in Arabidopsis [13,32]. Second, MsFTa shared several well characterized amino acid residues proven to be important for flowering acceleration according to the experiments of individual residue exchange or segment replacement. For example, in Arabidopsis, alteration of Tyr $85(\mathrm{Y} 85 \mathrm{H})$ changed FT functions in promoting flowering, indicating that the absolutely conserved Tyr 85 is a key residue determining the protein function as an activator of flowering [25]. FTs share a triad ending with residue asparagine $(\mathrm{N})$ at site 152 , followed by leucine (Figure 2). The crystal structure revealed that the triplet motif contacts the adjacent external loop, which facilitates access to FT interactors, such as the bZIP transcription factor FD and 14-3-3 proteins [24,33-35]. In Rosaceae species, mutations of the triad prevented the FT-like genes from promoting flowering [36]. Third, FTs have highly conserved motifs including a potential ligand-binding pocket and an external loop (Figure 2), both of which were proven to contribute to the functional specificity of FT $[24,33]$. Compared with other species, it seems that the external loop of legume plants is variant (Figure 2), implying varied conformation, possibly leading to flexible accessibility to a binding partner. Therefore, $M s F T a$, together with $F T$-like genes, specifies FT conformation by possessing identical exon/intron structure and highly conserved functional motifs.

FT-like orthologs from a variety of plant species have been shown to promote flowering when ectopically expressed [16,22,37-39]. In the model legume $M$. truncatula, reverse genetics revealed that the loss-of-function mutant of MtFTa1, the closest homolog of MsFTa, caused late flowering and MtFTa1 fully complemented fta1-1 defects [11]. Consistently, an allelic series of gain-of-function mutations in MtFTa1 caused by retroelement insertions exhibited a dominant early flowering phenotype [40,41], confirming its function in flowering regulation. It appears that similar to $F T, M s F T a$ from the perennial forage acts positively in flowering promotion. The notion is supported directly by the transgenic Arabidopsis and M. truncatula including both complementation and over-expression plants. Plants ectopically expressing $M s F T a$ in either wild type or $f t$ mutant of both plant species displayed a clear shift to early flowering (Figure $6 \mathrm{~d}-\mathrm{g}$ ). Statistically, the flowering time difference between the transgenic plants and the non-transgenic ones is significant $(p<0.05)$. Notably, compared with wild type, complementation plants flowered slightly late, suggesting that constitutive expression of $M s F T a$ partially rescued the flowering time of $f t$ mutants from Arabidopsis and barrel clover. These findings imply a minute functional distinction between MsFTa and its orthologs, FT and MtFTa1, in Arabidopsis and barrel clover, respectively. Application of the endogenous promoter of $M s F T a$ to produce transgenic alfalfa is undergoing. Moreover, generation of a loss of function mutant of $\mathrm{MsFTa}$ using the CRISPR-Cas9 strategy would be necessary to elucidate the endogenous function of MsFTa in alfalfa flowering regulation.

Given the detection of MsFTa in roots and stems, it is tempting to speculate that besides flowering promotion, MsFTa may participate in other development processes of alfalfa. FT orthologs have been documented to be involved in multiple functions like plant architecture [42], tuberization [15], and dormancy interruption [43]. The expression of MsFTa resembled FT orthologs in perennial plants, such as sweet cherry, loquat, leafy spurge and tea-oil tree, in which FT-like genes are expressed in diverse tissues, including mature leaves, floral buds and reproductive organs (flower organs and fruits) $[21,22,27,44]$. The expression divergence may be an important force in driving the functional division of $F T s$ in perennial plants, especially in species with multiple $F T$ paralogs $[13,14,16,22]$. As it is closely related to diploid legume M. truncatula, which has six identified FT-like genes, tetraploid alfalfa is more likely to have a similar number, or even more, MsFTa paralogs due to genome duplication. Long-term work of flowering regulation in alfalfa will be directed at paralogs of MsFTa.

\section{Materials and Methods}

\subsection{Plant Material and Growth Conditions}

Medicago sativa cv. 'Zhongmu No.1', bred by the Institute of Animal Science, the Chinese Academy of Agricultural Sciences, was used in this study. Seeds were germinated in regular soil in a 20 
cm diameter pot. Medicago truncatula Tnt1 insertion mutant fta1-1 (NF3307) was obtained from the Noble Research Institute (Ardomor, OK, USA). Arabidopsis thaliana seeds of ft-10 (CS9869) were from Arabidopsis Biological Research Center $(A B R C)$ and germinated in soil (vermiculite:soil = 1:3). All plants were grown in growth chambers at $21^{\circ} \mathrm{C}$ under a $16 / 8 \mathrm{~h}$ (light/dark) photoperiod.

\subsection{Cloning of MsFTa from Alfalfa}

Total RNA was extracted from 2-week-old alfalfa seedlings with TRIzol reagent (Sangon, Shanghai, China). After DNase I (Fermentas-MBI, Shenzhen, China) treatment, the first-strand cDNA was synthesized using Reverse Transcriptase III (Takara, Dalian, China). A DNA fragment was amplified by using the two degenerate primers designed based on FT-like genes in M. truncatula and sequenced. The obtained partial sequence of alfalfa $F T$-like gene was used to design primers for amplification of the full-length MsFTa by 3'-RACE and 5'-RACE (Invitrogen, Carlsbad, CA. USA) with the specific primers according to the manufacturer's instructions. PCR amplicons were purified after separated on $1 \%$ agarose gel and cloned into pMD19-T (TaKaRa, Dalian, China) and sequenced.

\subsection{Bioinformatic Analysis}

For FT homologous genes, Arabidopsis FT (At1g65480) was used as query sequence to search against the database EnsemblPlants (http://plants.ensembl.org/index.html). Phylogenetic analysis was performed with bootstrap trials of 1000 using DNAMAN version 7.0 (Lynnon Corporation, Quebec, QC, Canada), and sequence alignment was analyzed using the same software with default parameters. Isoelectric point (pI) and molecular weight (MW) values were predicted using ExPaSy (https:// prosite.expasy.org). The secondary structure of the protein was predicted using LYON-GERLAND PBIL (https://prabi.ibcp.fr); and the hydrophobic structure was predicted using ScanProsite (https: //prosite.expasy.org/scanprosite).

\subsection{Expression Analysis by RT-PCR}

For expression analysis in different tissues, roots, stems and leaves were harvested from 6-week-old alfalfa, while flowers were from 60-day-old plants. For the diurnal analysis, leaves were collected separately from 6-week-old alfalfa at zeitgeber time (ZT) 0, 2, 4, 6, 8, 10, 12, 14, 16, 18, 20, 22 and 24 time points, respectively. After extraction of total RNA, cDNA was prepared as described above. Semi-quantitative RT-PCR was carried out using gene-specific primers with MsActin serving as an internal control. The PCR product was analyzed in gel and images were captured using a Gel Image Analysis System FR-200A (Furi Science \& Technology, Shanghai, China). For quantitative real-time PCR, SYBR Premix EX Taq ${ }^{\mathrm{TM}}$ qPCR Kit (Takara, Dalian, China) was used according to the instruction with BIO-RAD CFX96 ${ }^{\mathrm{TM}}$ Real-Time System (BioRad, Hercules, CA, USA). The Arabidopsis Actin gene was used as a loading control. The sequence of the primers used in this study is listed in Table S2.

\subsection{Subcellular Localization Analysis}

For the subcellular localization analysis of MsFTa-GFP fusion protein in onion epidermal cells, the construct of MsFTa-GFP was performed as follows: the MsFTa ORF sequence was subcloned into the pA7-GFP (35S::GFP) expression vector [29] using the primers of FT-GF and FT-GR containing XhoI and SpeI restriction sites, respectively. The plasmid of the pA7-GFP and 35S::MsFTa-GFP fusion binary vector was then transformed into separate onion epidermal cells via particle bombardment (GJ-1000, Scientz Biotechnology, Ningbo, China). The subcellular localization of the transiently expressed MsFTa-GFP fusion protein was imaged using a confocal laser-scanning microscope (Olympus FV500, Tokyo, Japan). 


\subsection{Plasmid construct and plant transformation}

For generation of the transgenic Arabidopsis plants expressing 35S::MsFTa, first, a binary vector harboring 35S::MsFTa was constructed by amplifying MsFTa ORF with primers named pBI-FTa-f and pBI-FTa-r, and the sequence-confirmed MsFTa was introduced into the pBI121 vector (Clontech, Palo Alto, CA, USA) using Xba I and Sma I restriction sites. Secondly, the 35S::MsFTa plasmid was transformed into Agrobacterium tumefaciens strain GV3101 by electroporation, and Arabidopsis transformation was performed via the floral-dip method. T1 Arabidopsis seeds were screened on a MS medium containing $40 \mathrm{mg} \cdot \mathrm{L}^{-1}$ kanamycin, and T2 lines confirmed by semi-quantitative RT-PCR were used in this study for MsFTa expression and phenotypic analysis. For Medicago transformation, explants from 4-week-old plants (R108 and fta1-1) growing under LDs were cocultivated with the agrobacterium containing the construct of 35S::MsFTa according to the Medicago handbook [45]. Kanamycin of $40 \mathrm{mg} \cdot \mathrm{L}^{-1}$ was used to select transformant plants.

\section{Conclusions}

In this study, the FT homolog from alfalfa, one of the most important perennial forages, was isolated and characterized. MsFTa has highly identical sequence composition, especially exon/intron boundary and functional domains, with FTs in a variety of plant species. The diurnally regulated MsFTa is expressed preferentially in leaves and floral buds. Functionally, MsFTa accelerated flowering in Arabidopsis and the annual legume M. truncatula, indicating that the novel alfalfa FT acts as a flowering promoter.

Supplementary Materials: Supplementary materials can be found at http://www.mdpi.com/1422-0067/20/8/1968/ s1.

Author Contributions: J.M.K. and T.J.Z. designed the experiments; T.G. and W.D. performed experiments; R.C.L. analyzed the data; Q.C.Y. and Z.W. wrote the paper.

Funding: This research was funded by the Agricultural Science and Technology Innovation Program (ASTIP-IAS-TS-14), the Fundamental Research Funds for Central Non-profit Scientific Institution (2017ywf-2d-3) and National Natural Science Foundation of China (31772663).

Acknowledgments: We are grateful to Longxi Yu for critically reading the manuscript and the anonymous reviewers for the constructive comments.

Conflicts of Interest: The authors declare that they have no conflict of interest.

\section{References}

1. Blazquez, M.A.; Green, R.; Nilsson, O.; Sussman, M.R.; Weigel, D. Gibberellins promote flowering of arabidopsis by activating the LEAFY promoter. Plant Cell 1998, 10, 791-800. [CrossRef]

2. Huq, E.; Tepperman, J.M.; Quail, P.H. GIGANTEA is a nuclear protein involved in phytochrome signaling in Arabidopsis. Proc. Natl. Acad. Sci. USA 2000, 97, 9789-9794. [CrossRef] [PubMed]

3. Okamuro, J.K.; Szeto, W.; Lotys-Prass, C.; Jofuku, K.D. Photo and hormonal control of meristem identity in the Arabidopsis flower mutants apetala2 and apetala1. Plant Cell 1997, 9, 37-47. [CrossRef]

4. Wu, G.; Park, M.Y.; Conway, S.R.; Wang, J.W.; Weigel, D.; Poethig, R.S. The sequential action of miR156 and miR172 regulates developmental timing in Arabidopsis. Cell 2009, 138, 750-759. [CrossRef] [PubMed]

5. Huang, T.; Bohlenius, H.; Eriksson, S.; Parcy, F.; Nilsson, O. The mRNA of the Arabidopsis gene FT moves from leaf to shoot apex and induces flowering. Science 2005, 309, 1694-1696. [CrossRef] [PubMed]

6. Putterill, J.; Robson, F.; Lee, K.; Simon, R.; Coupland, G. The CONSTANS gene of Arabidopsis promotes flowering and encodes a protein showing similarities to zinc finger transcription factors. Cell 1995, 80, 847-857. [CrossRef]

7. Samach, A.; Onouchi, H.; Gold, S.E.; Ditta, G.S.; Schwarz-Sommer, Z.; Yanofsky, M.F.; Coupland, G. Distinct roles of CONSTANS target genes in reproductive development of Arabidopsis. Science 2000, 288, 1613-1616. [CrossRef] [PubMed] 
8. Hecht, V.; Foucher, F.; Ferrandiz, C.; Macknight, R.; Navarro, C.; Morin, J.; Vardy, M.E.; Ellis, N.; Beltran, J.P.; Rameau, C.; et al. Conservation of Arabidopsis flowering genes in model legumes. Plant Physiol. 2005, 137, 1420-1434. [CrossRef]

9. Ruelens, P.; de Maagd, R.A.; Proost, S.; Theissen, G.; Geuten, K.; Kaufmann, K. FLOWERING LOCUS C in monocots and the tandem origin of angiosperm-specific MADS-box genes. Nat. Commun. 2013, 4, 2280. [CrossRef]

10. Wong, A.C.; Hecht, V.F.; Picard, K.; Diwadkar, P.; Laurie, R.E.; Wen, J.; Mysore, K.; Macknight, R.C.; Weller, J.L. Isolation and functional analysis of CONSTANS-LIKE genes suggests that a central role for CONSTANS in flowering time control is not evolutionarily conserved in Medicago truncatula. Front. Plant Sci. 2014, 5, 486. [CrossRef]

11. Laurie, R.E.; Diwadkar, P.; Jaudal, M.; Zhang, L.; Hecht, V.; Wen, J.; Tadege, M.; Mysore, K.S.; Putterill, J.; Weller, J.L.; et al. The Medicago FLOWERING LOCUS T homolog, MtFTa1, is a key regulator of flowering time. Plant Physiol. 2011, 156, 2207-2224. [CrossRef] [PubMed]

12. Tamaki, S.; Matsuo, S.; Wong, H.L.; Yokoi, S.; Shimamoto, K. Hd3a protein is a mobile flowering signal in rice. Science 2007, 316, 1033-1036. [CrossRef]

13. Wang, Z.; Zhou, Z.; Liu, Y.; Liu, T.; Li, Q.; Ji, Y.; Li, C.; Fang, C.; Wang, M.; Wu, M.; et al. Functional evolution of phosphatidylethanolamine binding proteins in soybean and Arabidopsis. Plant Cell 2015, 27, 323-336. [CrossRef] [PubMed]

14. Komiya, R.; Yokoi, S.; Shimamoto, K. A gene network for long-day flowering activates RFT1 encoding a mobile flowering signal in rice. Development 2009, 136, 3443-3450. [CrossRef]

15. Navarro, C.; Abelenda, J.A.; Cruz-Oro, E.; Cuellar, C.A.; Tamaki, S.; Silva, J.; Shimamoto, K.; Prat, S. Control of flowering and storage organ formation in potato by FLOWERING LOCUS T. Nature 2011, 478, 119-122. [CrossRef] [PubMed]

16. Niu, L.; Fu, C.; Lin, H.; Wolabu, T.W.; Wu, Y.; Wang, Z.Y.; Tadege, M. Control of floral transition in the bioenergy crop switchgrass. Plant Cell Environ. 2016, 39, 2158-2171. [CrossRef] [PubMed]

17. Skot, L.; Sanderson, R.; Thomas, A.; Skot, K.; Thorogood, D.; Latypova, G.; Asp, T.; Armstead, I. Allelic variation in the perennial ryegrass FLOWERING LOCUS T gene is associated with changes in flowering time across a range of populations. Plant Physiol. 2011, 155, 1013-1022. [CrossRef]

18. Hsu, C.Y.; Adams, J.P.; Kim, H.; No, K.; Ma, C.; Strauss, S.H.; Drnevich, J.; Vandervelde, L.; Ellis, J.D.; Rice, B.M.; et al. FLOWERING LOCUS T duplication coordinates reproductive and vegetative growth in perennial poplar. Proc. Natl. Acad. Sci. USA 2011, 108, 10756-10761. [CrossRef]

19. Song, G.Q.; Walworth, A.; Zhao, D.; Jiang, N.; Hancock, J.F. The Vaccinium corymbosum FLOWERING LOCUS T-like gene (VcFT): A flowering activator reverses photoperiodic and chilling requirements in blueberry. Plant Cell Rep. 2013, 32, 1759-1769. [CrossRef]

20. Trankner, C.; Lehmann, S.; Hoenicka, H.; Hanke, M.V.; Fladung, M.; Lenhardt, D.; Dunemann, F.; Gau, A.; Schlangen, K.; Malnoy, M.; et al. Over-expression of an FT-homologous gene of apple induces early flowering in annual and perennial plants. Planta 2010, 232, 1309-1324. [CrossRef]

21. Hao, X.; Chao, W.; Yang, Y.; Horvath, D. Coordinated Expression of FLOWERING LOCUS T and DORMANCY ASSOCIATED MADS-BOX-Like Genes in Leafy Spurge. PLoS ONE 2015, 10, e0126030. [CrossRef]

22. Yarur, A.; Soto, E.; Leon, G.; Almeida, A.M. The sweet cherry (Prunus avium) FLOWERING LOCUS T gene is expressed during floral bud determination and can promote flowering in a winter-annual Arabidopsis accession. Plant Reprod. 2016, 29, 311-322. [CrossRef] [PubMed]

23. Varkonyi-Gasic, E.; Moss, S.M.; Voogd, C.; Wang, T.; Putterill, J.; Hellens, R.P. Homologs of FT, CEN and FD respond to developmental and environmental signals affecting growth and flowering in the perennial vine kiwifruit. New Phytol. 2013, 198, 732-746. [CrossRef]

24. Ahn, J.H.; Miller, D.; Winter, V.J.; Banfield, M.J.; Lee, J.H.; Yoo, S.Y.; Henz, S.R.; Brady, R.L.; Weigel, D. A divergent external loop confers antagonistic activity on floral regulators FT and TFL1. EMBO J. 2006, 25, 605-614. [CrossRef]

25. Hanzawa, Y.; Money, T.; Bradley, D. A single amino acid converts a repressor to an activator of flowering. Proc. Natl. Acad. Sci. USA 2005, 102, 7748-7753. [CrossRef] [PubMed]

26. Li, C.; Luo, L.; Fu, Q.; Niu, L.; Xu, Z.F. Isolation and functional characterization of JcFT, a FLOWERING LOCUS T (FT) homologous gene from the biofuel plant Jatropha curcas. BMC Plant Biol. 2014, 14, 125. [CrossRef] [PubMed] 
27. Lei, H.; Su, S.; Ma, L.; Wen, Y.; Wang, X. Molecular cloning and functional characterization of CoFT1, a homolog of FLOWERING LOCUS T (FT) from Camellia oleifera. Gene 2017, 626, 215-226. [CrossRef]

28. Nelson, M.N.; Ksiazkiewicz, M.; Rychel, S.; Besharat, N.; Taylor, C.M.; Wyrwa, K.; Jost, R.; Erskine, W.; Cowling, W.A.; Berger, J.D.; et al. The loss of vernalization requirement in narrow-leafed lupin is associated with a deletion in the promoter and de-repressed expression of a Flowering Locus T (FT) homologue. New Phytol. 2017, 213, 220-232. [CrossRef]

29. Li, Z.; Long, R.; Zhang, T.; Wang, Z.; Zhang, F.; Yang, Q.; Kang, J.; Sun, Y. Molecular cloning and functional analysis of the drought tolerance gene MsHSP70 from alfalfa (Medicago sativa L.). J. Plant Res. 2017, 130, 387-396. [CrossRef]

30. Lifschitz, E.; Eshed, Y. Universal florigenic signals triggered by FT homologues regulate growth and flowering cycles in perennial day-neutral tomato. J. Exp. Bot. 2006, 57, 3405-3414. [CrossRef]

31. Andres, F.; Coupland, G. The genetic basis of flowering responses to seasonal cues. Nat. Rev. Genet. 2012, 13, 627-639. [CrossRef]

32. Ho, W.W.; Weigel, D. Structural features determining flower-promoting activity of Arabidopsis FLOWERING LOCUS T. Plant Cell 2014, 26, 552-564. [CrossRef]

33. Abe, M.; Kobayashi, Y.; Yamamoto, S.; Daimon, Y.; Yamaguchi, A.; Ikeda, Y.; Ichinoki, H.; Notaguchi, M.; Goto, K.; Araki, T. FD, a bZIP protein mediating signals from the floral pathway integrator FT at the shoot apex. Science 2005, 309, 1052-1056. [CrossRef] [PubMed]

34. Kaneko-Suzuki, M.; Kurihara-Ishikawa, R.; Okushita-Terakawa, C.; Kojima, C.; Nagano-Fujiwara, M.; Ohki, I.; Tsuji, H.; Shimamoto, K.; Taoka, K.I. TFL1-Like Proteins in Rice Antagonize Rice FT-Like Protein in Inflorescence Development by Competition for Complex Formation with 14-3-3 and FD. Plant Cell Physiol. 2018, 59, 458-468. [CrossRef]

35. Taoka, K.; Ohki, I.; Tsuji, H.; Furuita, K.; Hayashi, K.; Yanase, T.; Yamaguchi, M.; Nakashima, C.; Purwestri, Y.A.; Tamaki, S.; et al. 14-3-3 proteins act as intracellular receptors for rice Hd3a florigen. Nature 2011, 476, 332-335. [CrossRef]

36. Wang, Z.; Yang, R.; Devisetty, U.K.; Maloof, J.N.; Zuo, Y.; Li, J.; Shen, Y.; Zhao, J.; Bao, M.; Ning, G. The Divergence of Flowering Time Modulated by FT/TFL1 Is Independent to Their Interaction and Binding Activities. Front. Plant Sci. 2017, 8, 697. [CrossRef]

37. Pin, P.A.; Nilsson, O. The multifaceted roles of FLOWERING LOCUS T in plant development. Plant Cell Environ. 2012, 35, 1742-1755. [CrossRef]

38. Voogd, C.; Brian, L.A.; Wang, T.; Allan, A.C.; Varkonyi-Gasic, E. Three FT and multiple CEN and BFT genes regulate maturity, flowering, and vegetative phenology in kiwifruit. J. Exp. Bot. 2017, 68, 1539-1553. [CrossRef] [PubMed]

39. Wolabu, T.W.; Zhang, F.; Niu, L.; Kalve, S.; Bhatnagar-Mathur, P.; Muszynski, M.G.; Tadege, M. Three FLOWERING LOCUS T-like genes function as potential florigens and mediate photoperiod response in sorghum. New Phytol. 2016, 210, 946-959. [CrossRef] [PubMed]

40. Jaudal, M.; Yeoh, C.C.; Zhang, L.; Stockum, C.; Mysore, K.S.; Ratet, P.; Putterill, J. Retroelement insertions at the Medicago FTa1 locus in spring mutants eliminate vernalisation but not long-day requirements for early flowering. Plant J. 2013, 76, 580-591. [CrossRef]

41. Yeoh, C.C.; Balcerowicz, M.; Zhang, L.; Jaudal, M.; Brocard, L.; Ratet, P.; Putterill, J. Fine mapping links the FTa1 flowering time regulator to the dominant spring1 locus in Medicago. PLoS ONE 2013, 8, e53467. [CrossRef] [PubMed]

42. Lifschitz, E.; Eviatar, T.; Rozman, A.; Shalit, A.; Goldshmidt, A.; Amsellem, Z.; Alvarez, J.P.; Eshed, Y. The tomato FT ortholog triggers systemic signals that regulate growth and flowering and substitute for diverse environmental stimuli. Proc. Natl. Acad. Sci. USA 2006, 103, 6398-6403. [CrossRef]

43. Rinne, P.L.; Welling, A.; Vahala, J.; Ripel, L.; Ruonala, R.; Kangasjarvi, J.; van der Schoot, C. Chilling of dormant buds hyperinduces FLOWERING LOCUS T and recruits GA-inducible 1,3-beta-glucanases to reopen signal conduits and release dormancy in Populus. Plant Cell 2011, 23, 130-146. [CrossRef] [PubMed] 
44. Reig, C.; Gil-Munoz, F.; Vera-Sirera, F.; Garcia-Lorca, A.; Martinez-Fuentes, A.; Mesejo, C.; Perez-Amador, M.A.; Agusti, M. Bud sprouting and floral induction and expression of FT in loquat [Eriobotrya japonica (Thunb.) Lindl.]. Planta 2017, 246, 915-925. [CrossRef] [PubMed]

45. Cosson, V.; Durand, P.; d'Erfurth, I.; Kondorosi, A.; Ratet, P. Medicago truncatula transformation using leaf explants. Methods Mol. Biol. 2006, 343, 115-127. [PubMed]

(C) 2019 by the authors. Licensee MDPI, Basel, Switzerland. This article is an open access article distributed under the terms and conditions of the Creative Commons Attribution (CC BY) license (http://creativecommons.org/licenses/by/4.0/). 